\title{
The Effects of Ankle Joint Position in Various Lower Limb Ground States on the Activation of the Shoulder and Trunk Muscles during Push-up Exercises
}

\author{
Gak Hwangbo, PT, PhD ${ }^{1)}$, Myung-Kwon Kim, PT, PhD²) \\ 1) Department of Physical Therapy, Daegu University \\ 2) Department of Physical Therapy, Youngsan University: Joonam-dong,Yangsan, Kyeongsangnam-do, \\ San 150, Republic of Korea. \\ TEL:+82 55-380-9367,FAX: +82 55-380-9305,E-mail: skybird-98@hanmail.net
}

\begin{abstract}
Purpose] This study was undertaken to quantify the effects of ankle joint position on unstable and stable surfaces during push up exercises on shoulder and trunk muscle activities. [Subjects] The study subjects were 45 healthy adults in their twenties with normal ranges of motion (ROM) and without disorders of the shoulder complex, musculoskeletal disease of the upper limbs, or low back pain. [Methods] Push-up exercises were performed under the following four conditions: (1) on an unstable surface created by placing a $65-\mathrm{cm}$ diameter exercise ball under the ankle joints in full dorsiflexion; (2) on an unstable surface created by placing a $65-\mathrm{cm}$ diameter exercise ball under the ankle joints in full plantarflexion; (3) on a stable surface created by placing a bench with a height of a $65-\mathrm{cm}$ under the ankle joints in full dorsiflexion; and (4) on a stable surface created by placing a bench with a height of 65$\mathrm{cm}$ under the ankle joints in full plantarflexion. To prevent bias, the exercise conditions were randomly arranged. [Results] Muscle activities were compared among the four different conditions. The erector spinae, rectus abdominis, external oblique abdominal, serratus anterior, deltoid middle fiber, and triceps brachii muscles showed significant differences among the four conditions. [Conclusion] These differences may have been resulted from increased trunk and shoulder muscle activations during push-up exercise with full ankle dorsiflexion on unstable ground.

Key words: Push-up, Unstable surface, Muscle activation
\end{abstract}

(This article was submitted Nov. 14, 2011, and was accepted Dec. 15, 2011)

\section{INTRODUCTION}

Of the closed chain exercises, push ups are extensively used for the rehabilitation of shoulder injuries. Push-up performance measures strength and endurance of the upper extremity and trunk muscles ${ }^{1)}$.

Regarding, push-up exercises on a stable surface, An et $\mathrm{al}^{2}{ }^{2)}$ reported that the positions of the palms, movement direction of the arms, position of the feet, and push up velocity in relation to gravity affect upper extremity joint pressure. Furthermore, the addition of surface instability in the form of a Swiss ball, wobble boards, and other labile surfaces, is an important consideration during shoulder rehabilitation. Exercise on a labile surface increases muscular demand to maintain postural stability ${ }^{3)}$, and it has been demonstrated that people use distinctive movement control behaviors to adapt to stable and unstable surfaces ${ }^{4}$. Garcia et al. ${ }^{5)}$ showed there is a consistent increase in selected trunk muscles during curl up on an exercise ball, and Naughton et al. ${ }^{6}$ showed that shoulder rehabilitation therapy using an unstable surface improves shoulder joint proprioception. In addition, Marshall and Murphy ${ }^{2}$ showed an increase in muscle activity when a Swiss ball was the primary base of support.

One common assumption is that an unstable surface places an increased demand on the neuromuscular system in the stabilization of articular joints made more unstable by the presence of a labile surface. The proposed benefits of training with this instability are improvements in joint proprioception and greater muscle activation ${ }^{7}$.

There have been many studies of push up exercises on stable and unstable surfaces. When performing push-up exercises with an object on the feet, people position their ankle joints in various ways. Some people dorsiflex or plantarflex their ankle joints during push-up exercises with an object under the feet. We are wondering whether ankle position during push-up exercises affects muscle activation in the shoulder and trunk. Few studies have investigated on muscle activation in the shoulder and trunk in the presence of lower limb instability and at different ankle joint positions during push-up exercises. Therefore in the present study, we attempted to quantify the effects of ankle joint position during push up exercises conducted with stable and unstable surfaces under the feet on shoulder and trunk muscle activaties. 


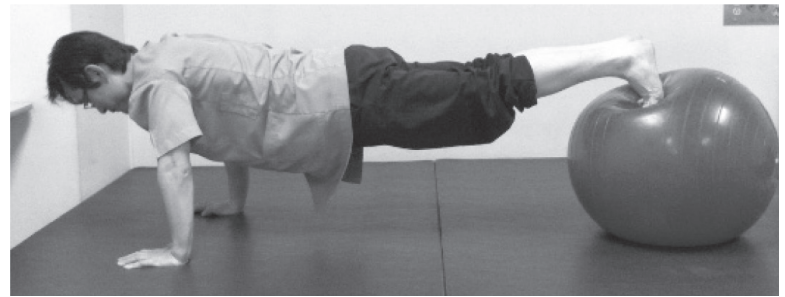

Fig. 1. Dorsiflexion - ball

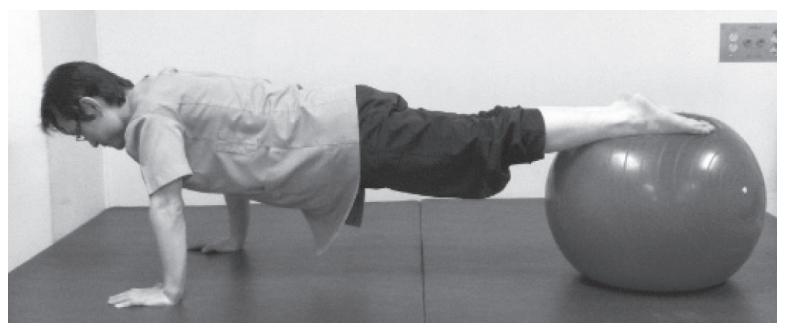

Fig. 3. Plantarflexion - ball

\section{SUBJECTS AND METHODS}

The subjects of this study were 45 healthy adults in their 20 s with normal ranges of motion (ROM), and without disorders of the shoulder complex or musculoskeletal disease in the upper limbs, or low back pain. Subjects were provided with a full explanation of the experimental procedures and all provided their written consent indicating their voluntary participation. The general characteristics of the subjects, 39 men and 6 women, were; age, $25.3 \pm 2.2$; height, $175.3 \pm$ $3.3 \mathrm{~cm}$; weight, $69.2 \pm 3.2 \mathrm{~kg}$; and BMI, $22.5 \pm 3.0$.

Push-up exercises were performed in the following manner. Cameras and personal computer monitors were used to provide visual information regarding scapular motion in the push-up position, and subjects were asked to observe their motions constantly using the computer monitors while performing the push-up exercises so they could perform accurate scapula protraction. While engaging in the push-up exercises, subjects were also asked to place their hands at shoulder width and to align the acromioclavicular joints and middle fingers.

The experiment was performed using the following four conditions.

Push-ups were performed: 1) on an unstable surface created by placing a $65-\mathrm{cm}$ diameter exercise ball under the ankle joints in full dorsiflexion (Fig. 1) ; 2) on an unstable surface created by placing a $65-\mathrm{cm}$ diameter exercise ball under the ankle joints in full plantarflexion (Fig. 2) ; 3) on a stable surface created by placing a bench of $65-\mathrm{cm}$ height under the ankle joints in full dorsiflexion (Fig. 3) ; and 4) on a stable surface created by placing a bench of $65-\mathrm{cm}$ height under the ankle joints in full plantarflexion(Fig. 4). Muscle activation measured three times, and the mean values were used in the analysis.

Push-ups were executed in the following manner.

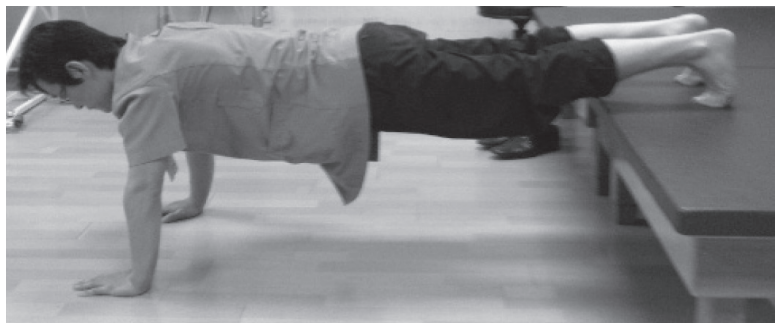

Fig. 2. Dorsiflexion - table

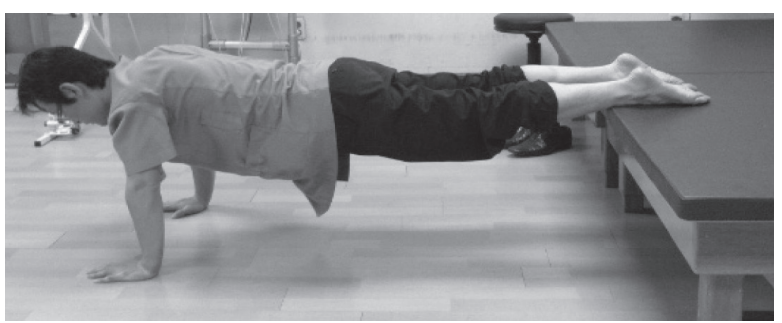

Fig. 4. Plantarflexion - table

Beginning in an upright position (when EMG collection was started) and held for $3 \mathrm{sec}$, then in an eccentric position, held for $3 \mathrm{sec}$, and finally in a concentric position, held for 3 sec. To prevent bias due to muscular fatigue of the shoulder stabilizers, participants performed the four exercises in a random order and a minimum of 3 minutes rest between exercises.

Electromyography (EMG) was performed after depilating the electrode attachment areas with a razor, removing the horny layer with sand paper, and cleansing with an alcohol swab. To measure muscle activations in the trunk, electrodes were attached to the erector spinae, the rectus abdominis, and external oblique abdominal muscle; the serratus anterior muscle, deltoid middle fiber muscle, pectoralis major muscle, and triceps brachii muscle were chosen as scapular stabilizers. ProComp InfinitiTM (Thought Technology Ltd., Canada) biofeedback software was used to measure muscle activations. The surface electrodes used were composed of three electrodes. The frequency range of the EMG signal was between 20 and $500 \mathrm{~Hz}$, and the sampling frequency was $1024 \mathrm{~Hz}$. Root mean square values for each muscle were measured for five seconds in the anatomical position. Relative muscle contractions were calculated with respect to the mean EMG of the middle three seconds, excluding the measurements of the first second and the last second. Muscle activities resulting from one push-up were expressed as relative muscle contractions in \%RVC. Measured data were analyzed by one-way ANOVA in SPSS for Windows (version 12.0) and the activities of shoulder stabilizer muscles and trunk muscles were compared among the conditions. Statistical significance was accepted for $\mathrm{p}$ values of $<$ 0.05 . 
Table 1 . Mean muscle activities during the four push up exercises

unit: \%RVC

\begin{tabular}{lcccc}
\hline Muscle & Dorsiflexion - ball & Dorsiflexion - table & Plantarflexion - ball & Plantarflexion - table \\
\hline ES* $^{*}$ & $394.1 \pm 38.8^{\mathrm{a}}$ & $227.5 \pm 29.0^{\mathrm{b}}$ & $263.7 \pm 35.5^{\mathrm{b}}$ & $200.2 \pm 24.6^{\mathrm{b}}$ \\
RA* $^{*}$ & $1293.1 \pm 235.9^{\mathrm{a}}$ & $437.3 \pm 47.5^{\mathrm{b}}$ & $611.9 \pm 109.4^{\mathrm{b}}$ & $296.8 \pm 27.0^{\mathrm{b}}$ \\
EOA* $^{*}$ & $1240.5 \pm 397.8^{\mathrm{a}}$ & $430.3 \pm 100.9^{\mathrm{b}}$ & $504.2 \pm 100.1^{\mathrm{a}}$ & $271.9 \pm 51.7^{\mathrm{b}}$ \\
SA $^{*}$ & $529.8 \pm 56.7^{\mathrm{a}}$ & $364.6 \pm 40.1^{\mathrm{b}}$ & $414.6 \pm 41.5^{\mathrm{a}}$ & $326.3 \pm 35.9^{\mathrm{b}}$ \\
TRI $^{*}$ & $29620.2 \pm 2659.1^{\mathrm{a}}$ & $20850.2 \pm 2286.4^{\mathrm{b}}$ & $22447.2 \pm 2338.4^{\mathrm{a}}$ & $18623.8 \pm 2088.3^{\mathrm{b}}$ \\
DMF* $^{*}$ & $17239.9 \pm 2523.9^{\mathrm{a}}$ & $11289.1 \pm 1158.5^{\mathrm{a}}$ & $12243.9 \pm 1882.9^{\mathrm{a}}$ & $9783.7 \pm 1514.9^{\mathrm{b}}$ \\
PM & $6080.2 \pm 506.1$ & $5077.1 \pm 359.0$ & $5929.6 \pm 455.7$ & $5933.2 \pm 1911.2$ \\
\hline
\end{tabular}

Dorsiflexion - ball: Push-up exercise was performed on an unstable surface created by placing a 65 -cm diameter exercise ball under the ankle joints in full dorsiflexion. Dorsiflexion - table: Push-up exercise was performed on a stable surface created by placing a bench of $65-\mathrm{cm}$ height under the ankle joints in full dorsiflexion. Plantarflexion - ball: Push-up exercise was performed on an unstable surface created by placing a $65-\mathrm{cm}$ diameter exercise ball under the ankle joints in full plantarflexion. Plantarflexion - table: Push-up exercise was performed on a stable surface created by placing a bench of $65-\mathrm{cm}$ height under the ankle joints in full plantarflexion ES, erector spinae muscle; RA, rectus abdominis muscle; EOA, external oblique abdominal muscle; SA, serratus anterior muscle; DMF, deltoid middle fiber muscle; PM, pectoralis major muscle; TB, triceps brachii muscle

NOTE. Values are means \pm SEs. Values with different superscripts in the same column differ significantly $(\mathrm{p}<0.05)$ according to Turkey's test

\section{RESULTS}

Mean erector spinae muscle activity was $394.1 \pm 38.8$ under the Dorsiflexion - ball condition, $227.5 \pm 29.0$ under the Dorsiflexion - table condition, $263.7 \pm 35.5$ under the Plantarflexion - ball condition, and $200.2 \pm 24.6$ under the Plantarflexion - table condition, and these values were significantly different. Post hoc testing showed a significant difference between the Dorsiflexion - ball and the Dorsiflexion - table results, between the Dorsiflexion - ball and the Plantarflexion - ball results, and between the Dorsiflexion - ball and the Plantarflexion - table results. Mean rectus abdominis muscle activity was $1293.1 \pm 235.9$ under the Dorsiflexion - ball condition, $437.3 \pm 47.5$ under the Dorsiflexion - table condition, $611.9 \pm 109.4$ under the Plantarflexion - ball condition, and $296.8 \pm 27.0$ under the Plantarflexion - table condition, and these values were also significantly different. Post-hoc testing showed a significant difference between the Dorsiflexion - ball and the Dorsiflexion - table results, between the Dorsiflexion - ball and the Plantarflexion - ball results, and between the Dorsiflexion - ball and the Plantarflexion - table results. Mean external oblique abdominal muscle activity was $1240.5 \pm$ 397.8 under the Dorsiflexion - ball condition, $430.3 \pm 100.9$ under the Dorsiflexion - table condition, 504.2 \pm 100.1 under the Plantarflexion - ball condition, and 271.9 \pm 51.7 under the Plantarflexion - table condition, which were also significantly different. The post hoc test showed a significant difference between the Dorsiflexion - ball and the Dorsiflexion - table results, and between the Dorsiflexion - ball and the Plantarflexion - table results. Mean serratus anterior muscle activity was $529.8 \pm 56.7$ under the Dorsiflexion ball condition, $364.6 \pm 40.1$ under the Dorsiflexion - table condition, $414.6 \pm 41.5$ under the Plantarflexion - ball condition, and $326.3 \pm 35.9$ under the Plantarflexion - table condition, which were also significantly different. The post hoc test showed a significant difference between the
Dorsiflexion - ball and the Dorsiflexion - table results, and between the Dorsiflexion - ball and the Plantarflexion table results. Mean deltoid middle fiber muscle activity was $17239.9 \pm 2523.9$ under the Dorsiflexion - ball condition, $11289.1 \pm 1158.5$ under the Dorsiflexion - table condition, $12243.9 \pm 1882.9$ under the Plantarflexion - ball condition, and $9783.7 \pm 1514.9$ under the Plantarflexion - table condition, and these too were significantly different. The post hoc test showed a significant difference between the Dorsiflexion - ball and the Plantarflexion - table results (Table 1).

\section{DISCUSSION}

In this study, volunteers performed push-up exercises on a stable surface and on an unstable surface with their feet at a higher level than their hands. The effects of ankle joint position on muscle activations in the upper limbs and trunk were then were analyzed. Muscle activity analysis showed significant differences among the individual conditions for the erector spinae muscle, rectus abdominis muscle, external oblique abdominal muscle, serratus anterior muscle, deltoid middle fiber muscle, and triceps brachii muscle. However, the pectoralis major muscle did not show a significant difference. Furthermore, changes in erector spinae muscle, rectus abdominis muscle, external oblique abdominal muscle, serratus anterior muscle, deltoid middle fiber muscle, and triceps brachii muscle activities in the trunk and upper extremity during the push-up exercise were found to be significantly different among the four conditions used.

The ankle joint position affects the distance between the foot/floor contact area. Also, surface instability increases upper extremity and trunk muscle activity. Although we did not measure the position of the center of mass, we presume that subjects' centers of mass were further removed from the foot/floor contact region when ankles were in dorsiflexion, as compared with plantarflexion. The pectoralis 
major muscle showed no significant activity differences among the four push-up exercises, possibly because of the difference between joints passing two muscles and associated movement. The pectoralis major performs a role in primary movement, but was found to be minimally affected by stability changes in the present study. There is greater redundancy in the motor control of muscles crossing the anterior shoulder. The shoulder joint is stabilized by the biceps brachii, deltoid, and rotator cuff muscles and shoulder adduction torque is also created by muscles other than the pectoralis major. The shoulder also has a smaller range of motion than the elbow joint. The pectoralis major is a single joint muscle, but the triceps brachii is a two joint muscle with stability and movement demands at the elbow and the shoulder, which possibly resulted in the dramatic changes in muscle activity seen in the present study. The triceps brachii has a mechanical advantage relative to the length of the forearm, as was observed by Lehman et al. ${ }^{7)}$.

\section{ACKNOWLEDGEMENT}

This research was supported by a Daegu University Research Grant, 2012.

\section{REFERENCES}

1) Cogley RM, Archambault TA, Fibeger JF, et al.: Comparison of muscle activation using various hand positions during the push-up exercise. J Strength Cond Res, 2005, 19: 628-633. [Medline]

2) An KN, Korinek SL, Kilpela T, et al.: Kinematic and kinetic analysis of push-up exercise. Biomed Sci Instrum, 1990, 26: 53-57. [Medline]

3) Marshall P, Murphy B: Changes in muscle activity and perceived exertion during exercises performed on a swiss ball. Appl Physiol Nutr Metab, 2006, 31: 376-383. [Medline] [CrossRef]

4) Franklin DW, Osu R, Burdet E, et al.: Adaption to stable and unstable dynamics achieved by combined impedance control and inverse dynamic model. Neurophysiol, 2003, 90: 3270-3282. [CrossRef]

5) Vera-Garcia FJ, Grenier SG, McGill SM, et al.: Abdominal muscle response during curl ups on both stable and labile surfaces. Phys Ther, 2000, 80: 564-569. [Medline]

6) Naughton J, Adams R, Maher C: Upper-body wobble board training effects on the post dislocation shoulder. Phys Ther Sport, 2005, 6: 31-37. [CrossRef]

7) Lehman GJ, MacMillan B, Maclntyre I, et al.: Shoulder muscle EMG activity during push up variations on and off a swiss ball. Dyn Med, 2006, 5: 7. [Medline] [CrossRef] 